\title{
The Calcination Temperature on the Electrochemical Properties of MnFe \\ IC Composite Oxides
}

\author{
Zhang Jiaheng \\ University of Changzhou, Jiangsu, Changzhou,213164, China \\ jiacheng@163.com
}

Keywords: Manganese metal, nickel iron talc, intercalation compound, carbon material, super capacitor.

\begin{abstract}
The super capacitor has the advantages of high power density, short charging time, long service life and so on. It has a great development potential in the field of energy. Continued growth in market share will further stimulate the super capacitor of $\mathrm{R} \& \mathrm{D}$, in order to meet the needs of commercial prepared has excellent capacity, high energy density and power density, good rate capability and cycle stability of electrode materials is a hot research at the present stage. Selection of $\mathrm{Mn}, \mathrm{Fe}$ as hydrotalcite plate elements, synthesis of dodecyl sodium sulfonate (SDOs) in layer hydrotalcite and after calcination at different temperatures formed metal oxide and carbon composite, study its application in the study on the properties of super capacitor electrode material.
\end{abstract}

\section{Introduction}

At present, some oxides of manganese, nickel, cobalt and vanadium in transition metals have been concerned by people in recent years. Manganese oxide $(\mathrm{MnOx})$ is considered to be a promising electrode material for super capacitor with low price and friendly environment. Manganese oxides are mainly derived from the pseudo capacitance, which is due to the reversible redox reactions, i.e., the conversion between Mn ions (III) / Mn (II), Mn (IV) / Mn (III), Mn (VI) / Mn (IV).

This experiment using hydrotalcite interlaminar to insert organic matter, after roasting method of one step formed metal oxide and carbon composite, for supercapacitor electrode materials research, to electric double layer capacitance and pseudocapacitance collaborative storage, material with large specific capacity and improve the effect of stability. This chapter used Mn, Fe as hydrotalcite plate elements, synthesis of dodecyl sodium sulfonate (SDOs) in layer hydrotalcite and after calcination at different temperatures formed metal oxide and carbon composite, study its application in the study on the properties of super capacitor electrode material.

\section{Experimental content}

Preparation of MnFe-SDOS-LDH precursor. Mn2Fe-SDSO-LDH, Mn3Fe-SDSO-LDH (LDH-2, LDH-3, LDH-4, Mn4Fe-SDSO-LDH) were synthesized by co precipitation method in a series of twelve alkyl sulfonate (SDSO) intercalated with different metal ratios. Synthesis methods are as follows: the molar ratio of Mn (NO3) 2 (50phr), Fe (NO3) 3 - 9h2o and sodium dodecyl sulfonate solution to the free carbon dioxide in deionized water with salt solution, into four mouth bottle, $\mathrm{NaOH}$ in removal of carbon dioxide in deionized water with alkali solution, under the protection of nitrogen and keep stirring under, the alkali solution by low with salt solution, and the $\mathrm{pH}$ is controlled between $10+0.5$, suspension and after titration, continue to stir for $30 \mathrm{~min}$, 
crystallization, washing to neutral, dry spare.

Preparation of metal oxide / carbon composites using MnFe-SDOS-LDH as precursor. Mn hydrotalcite intercalation precursors were calcined in a tube furnace. Will tile of the products obtained in the alumina boat and placed in a tube furnace heating zone, under nitrogen atmosphere, according to 5 DEG $\mathrm{C}$ per minute of the heating rate up to 400 DEG C, 500 DEG C, 600 DEG C, 800 DEG C roasting $4 \mathrm{H}$.

Electrochemical test conditions. (1) Pre treatment of titanium mesh: the titanium mesh (200 mesh) cut into $1 \mathrm{~cm} \times 3 \mathrm{~cm}$ rectangular, cleaning and drying. (2) Working electrode preparation: the quality of a certain water talc powder, acetylene black, PVDF (3\%) according to the quality than 7:2:1 mixture, stir well and get the desired slurry, titanium mesh wash clean backup. The coating slurry to the titanium mesh, coated electrode into vacuum oven; drying for $12 \mathrm{~h}$, the dry electrodes on the tablet press press spare. Construction of three electrode system: $\mathrm{Hg} / \mathrm{HgO}$ as reference electrode, platinum electrode as auxiliary electrode, $\mathrm{KOH} 1 \mathrm{M}$ solution as electrolyte for electrochemical performance test. (3) The Shanghai Chen Hua CHI-650D electrochemical workstation were studied by cyclic voltammetry, using Arbin company in the United States MSTAT4+ constant current charging and discharging device for constant current charge discharge test.

\section{Results and Discussion}

\section{Morphology and structure characterization of the composite prepared with MnFe-SDOS-LDH as precursor}

1. XRD characterization. LDH-2, LDH-3, LDH-4, respectively, were synthesized by co precipitation method with different $\mathrm{Mn} / \mathrm{Fe}$ ratios. The samples were characterized by XRD. Characterization results as shown in Figure 1 sample at 3.4 degrees, 7.5 degrees, near 11.2 degree appeared characteristic diffraction peaks of hydrotalcite, corresponding to (003), (006), (009) crystal plane, and at 60 DEG (110) (113) crystal face diffraction peak, Ming synthesized samples with layered structure. Calculated by small angle (003) diffraction peaks and four in the layer structure of hydrotalcite interlayer spacing is about $2.6 \mathrm{~nm}$, LDHs layer thickness is $0.48 \mathrm{~nm}$, dodecyl sulfonate in the direction of the long axis for $193 \mathrm{~nm}$ can infer the dodecyl sulfonate to single-layer vertical to the direction of the plate arrangement in the interlayer, as shown in Figure 2

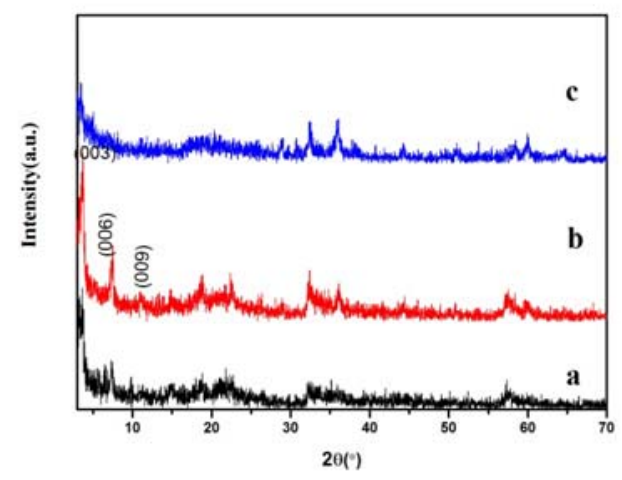

Fig. 1 XRD diffraction patterns of samples
(a) LDH-2
(b) LDH-3,
(c) $\mathrm{LDH}-4$ 


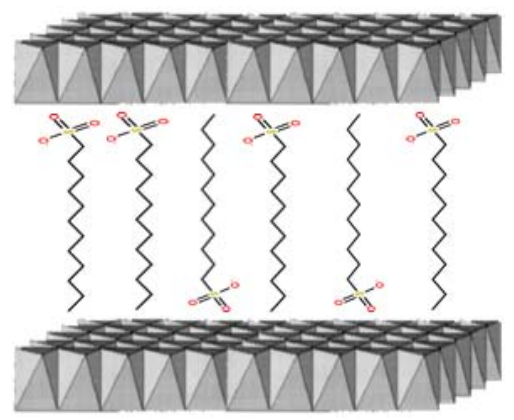

Fig.2 Structure model of dodecyl sulfonate-pillared

2. FT-IR. characterization. Will sample $1 \mathrm{dh}-2$, ldh-3 and ldh-4 for characterization of infrared spectrum, the results as shown in Figure 3, in $3450 \mathrm{~cm}-1$ have broad absorption peaks belonging to interlayer adsorption water stretching vibration and bending vibration. $2850-2920 \mathrm{~cm}-1$ wavenumber range appear $\mathrm{C}-\mathrm{H}$ stretching vibration absorption band, $1184 \mathrm{~cm}-1$ absorption peak is dodecyl sulfonate $\mathrm{s}$ asymmetric stretching vibration peak, show that dodecyl sulfonate into hydrotalcite between. The absorption peak of the absorption peak at $618 \mathrm{~cm}-1 \mathrm{~cm}-1532$ was attributed to the vibrational absorption peak at the lattice oxygen, which appeared at the peak of the flexural vibration of cm-1 OH- at the left and right.

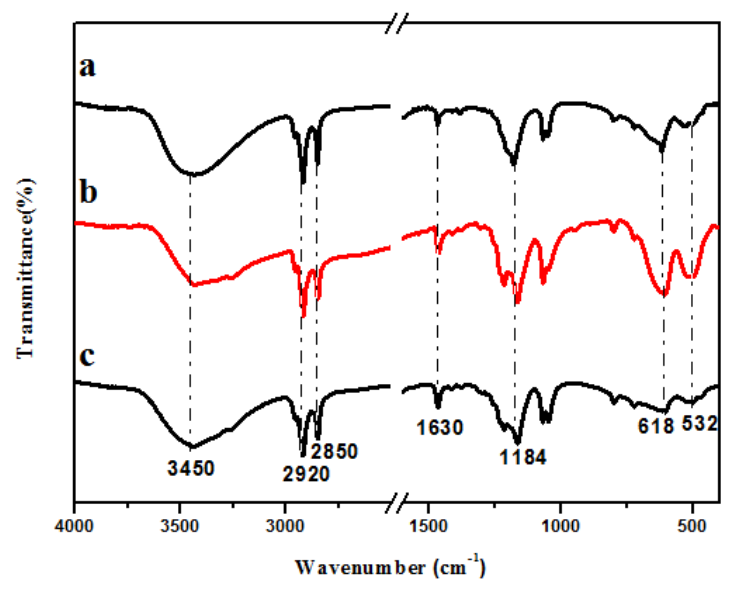

Fig.3 FT-IR spectrums of samples

(a) LDH-2, (b) LDH-3, (c) LDH-4

3. XRD characterization after calcination. The prepared samples were calcined at different temperatures by LDH-3. Of the calcined samples of XRD, hydrotalcite characteristic diffraction peaks disappear, at 34 degrees, 40 degrees, 58 degrees appear the characteristic diffraction peaks of MnO (jcpds No. 07-0230), at 35 DEG, 61 degrees appear the characteristic diffraction peaks of MnFe2O4 (jcpds No. 10-0319). Samples at 400 degrees C, 500 DEG C, 600 DEG C, 800 DEG C after calcination, hydrotalcite like precursor body into cubic $\mathrm{MnO}$ and $\mathrm{MnFe} 2 \mathrm{O} 4$ spinel structure, as shown in Figure 4. With increasing the calcination temperature, spinel phase diffraction peak intensity increasing, indicating that the composition of spinel phase increased with the increase of temperature. 


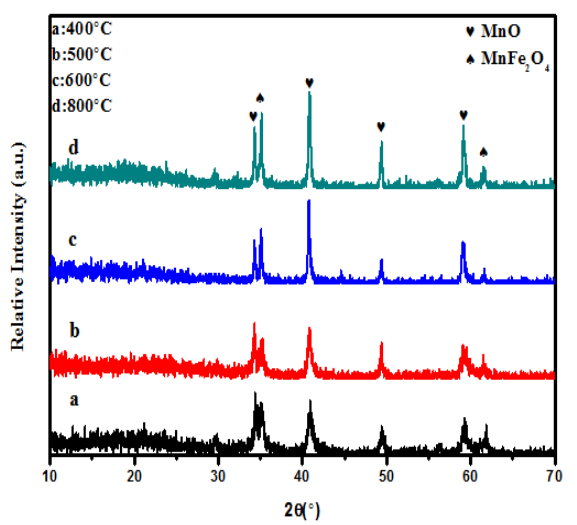

Fig.4 XRD patterns of $\mathrm{LDH}-3$ at the temperature of samples

(a) $400^{\circ} \mathrm{C}$, (b) $500^{\circ} \mathrm{C}$, (c) $600^{\circ} \mathrm{C}$, (d) $800^{\circ} \mathrm{C}$

4. SEM characterization after calcination. Through SEM observation after firing the morphology of the product, as shown in Figure 5, a, B, C, D were sample ldh-3 after 400 DEG C, 500 DEG C, 600 DEG C, scanning electron micrograph of 800 DEG C after calcination, from the figure can see that with the increase of calcination temperature, hydrotalcite flake like structure disappeared, product appeared nano particles, namely the generation of composite metal oxides, after 800 DEG $\mathrm{C}$ after calcination, generating massive spinel structure.
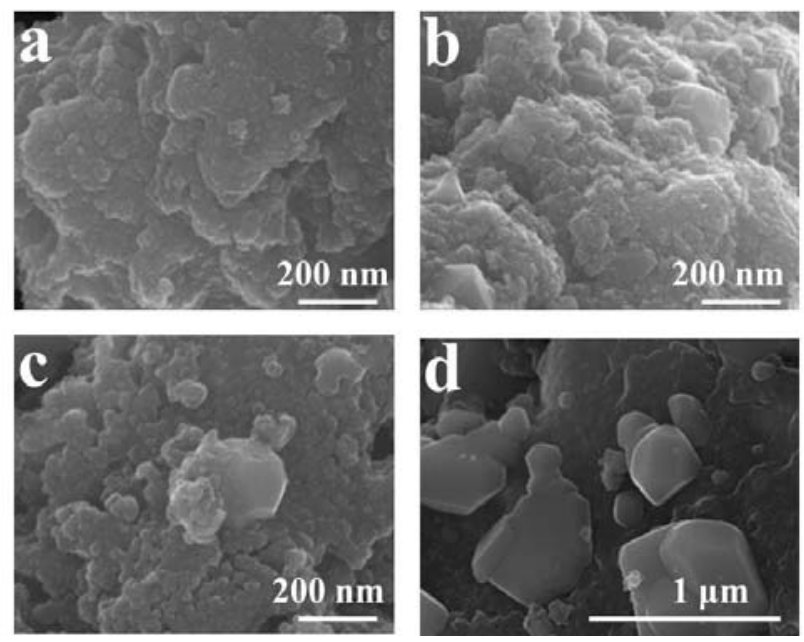

Fig. 5 SEM images of LDH-3 at the temperature of samples

(a) $400^{\circ} \mathrm{C}$, (b) $500^{\circ} \mathrm{C}$, (c) $600^{\circ} \mathrm{C}$, (d) $800^{\circ} \mathrm{C}$

Electrochemical characterization of composite materials using MnFe-SDOS-LDH as precursor

1. Cyclic voltammetry. Will Mn3Fe-SDSO-LDH calcined and acetylene black, PVDF (3\%) according to the quality than 7:2:1 mixed evenly coated on the Internet of titanium electrodes, vacuum drying for $12 \mathrm{~h}$, in the electrochemical workstation to characterize the electrochemical properties. Figure 7 shows the different calcination temperature of samples with different $\mathrm{KOH}$ electrolyte in $1 \mathrm{M}$ scanning rate $\mathrm{CV}$. A figure is 400 degrees $\mathrm{CV}$ after roasting $\mathrm{C}$ diagram, B diagram is 500 degrees $\mathrm{CV}$ after roasting $\mathrm{C}$ diagram, $\mathrm{C}$ diagram is 600 degrees $\mathrm{CV}$ after roasting $\mathrm{C}$ diagram, $\mathrm{D}$ diagram is 800 degrees $\mathrm{CV}$ after baking $\mathrm{C}$ diagram. In figure can see obvious redox peaks and $0.2 \mathrm{~V}-0.1 \mathrm{~V}$ oxidation reduction peak corresponds to a reaction $\mathrm{MnO}+\mathrm{OH}----\mathrm{Mn} 2 \mathrm{O} 3+\mathrm{e}-$. With the scan rate from $5 \mathrm{mV} \mathrm{S}-1$ increased to $20 \mathrm{mV} \mathrm{s}-1$, the redox peak current also increased. This is due to the high scan rate of reaction faster, from the figure a, B, C, D can be seen in the CV curve shape changes with scanning speed remained basically unchanged, indicating that the electrode in $\mathrm{KOH}$ 
electrolyte has good stability. With the increase of calcination temperature, the same sweep current strength corresponding to the speed reduction, show that the electrode material capacity decreases.
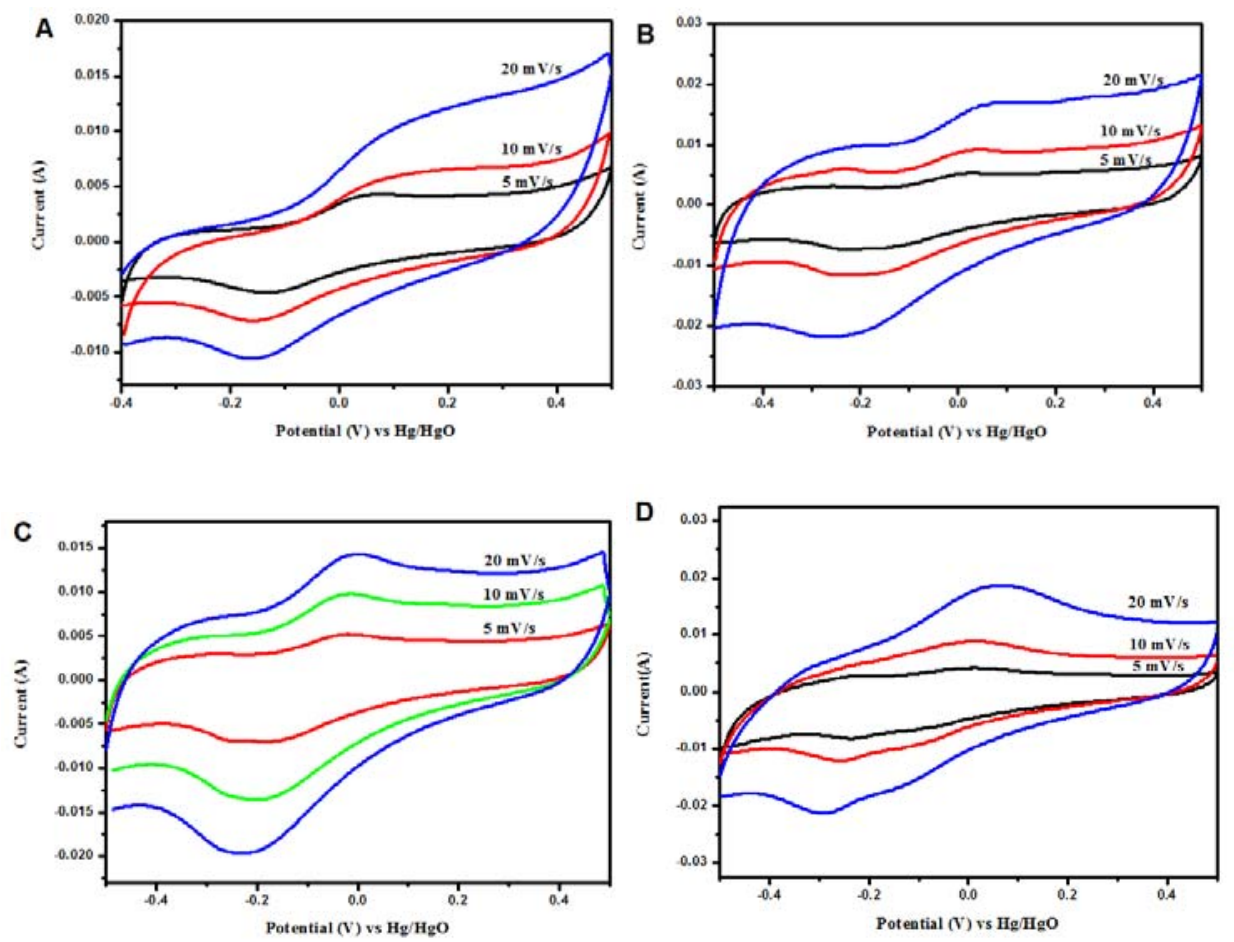

Fig.7 CV curves of LDO-3 at different calcination temperatures
(A) $400^{\circ} \mathrm{C},(\mathrm{B}) 500^{\circ} \mathrm{C}$,
(C) $600^{\circ} \mathrm{C},(\mathrm{D}) 800^{\circ} \mathrm{C}$

2. Charge discharge test and cycle performance test. The calcined samples LDO-2, LDO-3, LDO-4 were prepared as electrode materials, and the electrical properties were tested in the three electrode system, as shown in Figure 8, 9, 10. Figure $8 \mathrm{~A}$ for the sample LDO-2 at the current density of $1 \mathrm{~g}-1$ / A of the charge and discharge curve, according to the discharge time can calculate the specific capacity of the electrode, such as the formula 1 :

$$
\mathrm{C}=(\mathrm{I} \times \Delta \mathrm{t}) /(\mathrm{m} \times \triangle \mathrm{V})
$$

Where $\mathrm{I}$ is the discharge current (a), $\mathrm{m}$ for the active substance $(\mathrm{g})$, delta $\mathrm{T}$ ( $\mathrm{s}$ ) time for discharge, Delta $\mathrm{V}$ is the discharge voltage window (V), $\mathrm{C}$ for the specific electrode capacitance ( $\mathrm{f}$ g-1). From the figure, a can calculate the sample at 400 degrees C, 500 DEG C, 600 DEG C, 800 DEG C after roasting capacity were $60 \mathrm{f}$. g-1120 F. $100 \mathrm{fg}-1,80 \mathrm{fg}-1$, capacity is much higher in $500 \mathrm{C}$ that under the temperature of active sites exposed to more. Fig. B is a test chart for the cycle performance of samples LDH-2 at different firing temperatures, and the capacity retention of the electrode materials after cycle is investigated. From the chart to see at the beginning stage, the electrode specific capacitance increased trend, and then started to decline, which is due at the beginning of the charge and discharge, electrode materials were soaked in a relatively short time, not fully activated, with the reaction, the activity gradually improve. After a certain number of laps, lower electrode, the capacitance decreases gradually after 500 cycles the specific capacitance remained at $120 \mathrm{~F}$ g-1. 

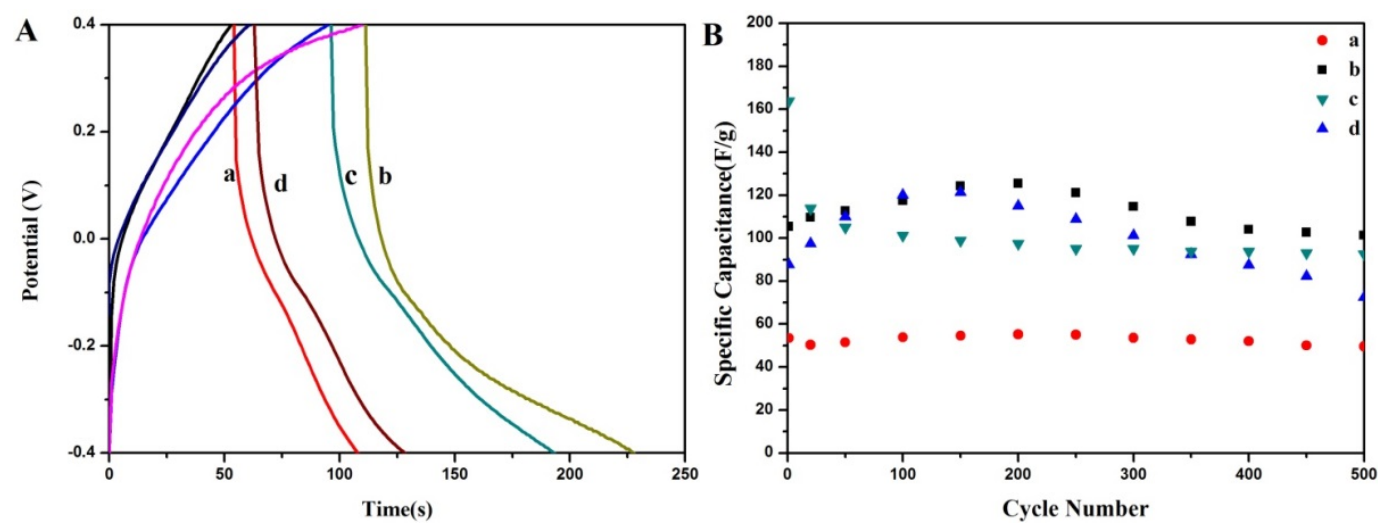

Fig.8 Electrochemical performance of LDH-2 at different calcination temperatures: (A) Galvanostatic charge-discharge curves , (B) cycle stability

(a) $400^{\circ} \mathrm{C}$, (b) $500^{\circ} \mathrm{C}$, (c) $600^{\circ} \mathrm{C}$, (d) $800^{\circ} \mathrm{C}$

\section{Summary}

(1) Choose easy in layer of organic matter dodecyl sulfonic acid sodium intercalation prepare SDOs in LDHs layers, through XRD, FT-IR characterization examines the proportion of different ferromanganese insertion layer hydrotalcite structure, layer spacing increased to $2.6 \mathrm{~nm}$, appear s characteristic absorption peak of infrared spectrum, thus indicating that SDOs was successfully inserted into LDHs.

(2) The insertion layer hydrotalcite after roasting formation of ferromanganese metal oxides and carbon composite, with the increase of calcination temperature, interlayer SDOs decomposition. In layered double hydroxides is changed to cubic phase composite oxide by the layered structure.

\section{Reference:}

[1] Zhang L, Zhang X, Shen L, Gao B, Hao L, Lu X, Zhang F, Ding B and Yuan C.Enhanced high-current capacitive behavior of graphene/CoAl-layered double hydroxide composites as electrode material for supercapacitors[J]. J. Power Sources, 2012, 199, 395-401

[2] Xu Z P, Li L, Cheng C Y, Ding R G, Zhou C H. High capacitance electrode materials based on layered double hydroxides prepared by non-aqueous precipitation[J].Appl. Clay. Sci, 2013, 74, 102-108

[3] Wang B, Zhang H, Evans D G, Duan X. Surface modification of layered double hydroxides and incorporation of hydrophobic organic compounds[J]. Mater Chem Phys, 2005, 92:190-196 